WHITE PAPER FOR THE

PLANETARY SCIENCE AND ASTROBIOLOGY DECADAL SURVEY, 2023 - 2032

\title{
Enabling and Enhancing Science Exploration Across the Solar System: Aerocapture Technology for SmallSat to Flagship Missions
}

\author{
Primary Author: Alex Austin ${ }^{1}$ \\ Jet Propulsion Laboratory/California Institute of Technology \\ Phone: 818-393-7521 \\ E-Mail: Alexander.Austin@jpl.nasa.gov
}

Co-Authors:

Gonçalo Afonso ${ }^{2}$

Samuel Albert ${ }^{3}$

Hisham Ali ${ }^{4}$

Antonella Alunni ${ }^{5}$

James Arnold ${ }^{6}$

Gilles Bailet $^{7}$

Patricia Beauchamp ${ }^{1}$

Alan Cassell ${ }^{5}$

Jim Cutts ${ }^{1}$

Rohan Deshmukh ${ }^{8}$

Robert Dillman ${ }^{9}$

Sarah D'Souza ${ }^{5}$

Soumyo Dutta ${ }^{9}$

Charles Edwards ${ }^{1}$

Donald Ellerby ${ }^{5}$

John Elliott ${ }^{1}$

Giusy Falcone ${ }^{10}$

Alberto Fedele ${ }^{11}$

Jay Feldman ${ }^{5}$

Anthony Freeman ${ }^{1}$

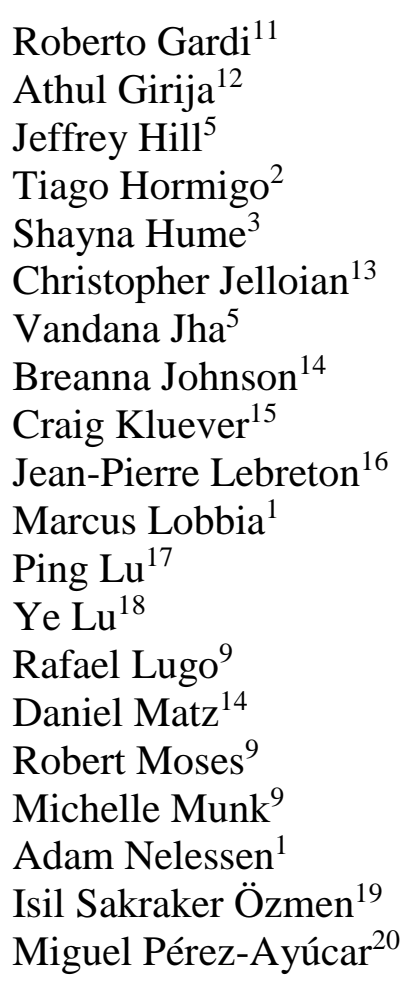

Richard Powell ${ }^{8}$

Zachary Putnam ${ }^{10}$

Jeremy Rea ${ }^{14}$

Sachin Alexander Reddy ${ }^{21}$

Thomas Reimer ${ }^{19}$

Sarag Saikia ${ }^{12}$

Kunio Sayanagi ${ }^{22}$

Stephan Schuster ${ }^{23}$

Jennifer Scully ${ }^{1}$

David Skulsky ${ }^{1}$

Ronald Sostaric ${ }^{14}$

Christophe Sotin ${ }^{1}$

Ben Tackett ${ }^{8}$

Ethiraj Venkatapathy ${ }^{5}$

Paul Wercinski ${ }^{5}$

Michael Wilder ${ }^{5}$

Michael Wright ${ }^{5}$

Cindy Young ${ }^{9}$

${ }^{1}$ Jet Propulsion Laboratory/California Institute of

${ }^{13}$ University of California, Los Angeles

Technology

${ }^{2}$ Spin.Works S.A

${ }^{3}$ University of Colorado Boulder

${ }^{4}$ Georgia Institute of Technology

${ }^{5}$ NASA Ames Research Center

${ }^{6}$ AMA, Inc. at NASA Ames Research Center

${ }^{7}$ University of Glasgow

${ }^{8}$ AMA, Inc. at NASA Langley Research Center

${ }^{9}$ NASA Langley Research Center

${ }^{10}$ University of Illinois at Urbana-Champaign

${ }^{11}$ C.I.R.A. Italian Aerospace Research Center

${ }^{12}$ Purdue University

\author{
${ }^{14}$ NASA Johnson Space Center \\ ${ }^{15}$ University of Missouri \\ ${ }^{16}$ French National Centre for Scientific Research \\ ${ }^{17}$ San Diego State University \\ ${ }^{18}$ Kent State University \\ ${ }^{19}$ Institute of Structures and Design - German \\ Aerospace Center (DLR) \\ ${ }^{20}$ Aurora Technology B.V. \\ ${ }^{21}$ Mullard Space Science Laboratory, University \\ College London \\ ${ }^{22}$ Hampton University \\ ${ }^{23}$ European Space Agency - ESTEC
}

Some of this work was carried out at the Jet Propulsion Laboratory, California Institute of Technology, under a contract with the National Aeronautics and Space Administration. 
Aerocapture technology provides a far-reaching capability for missions at destinations across the solar system, from SmallSat to Flagship class. It will enable a new class of SmallSat orbiters, allow high priority missions to carry more science payload to their destination, and open a new rapid transportation pathway to the outer solar system. Significant technology development and demonstration in the past decade has prepared high heritage aeroshell shapes to be ready to support the infusion of aerocapture into missions, particularly at the Ice Giants. In addition, research and focus on drag modulation control schemes has highlighted that this aerocapture trajectory control system can enable a new generation of small spacecraft science orbiters as secondary payloads on larger mission's launches under programs such as SIMPLEx, leading to increased science return at a fraction of the cost.

\section{Aerocapture Overview}

There are a great number of scientific questions to answer at locations throughout the solar system, but one fundamental challenge governs any mission: delivering the science payload to its destination. Astrodynamics dictates that a spacecraft must slow down significantly to enter orbit around a planetary body, or else only perform a single flyby. Historically, orbit insertion has been accomplished using a large propulsion system with a significant amount of propellant onboard the spacecraft, leading to "traditional" spacecraft with more than half of their mass being devoted to propulsion systems and propellants.

Aerocapture takes advantage of a naturally occurring resource at many planetary destinations, atmospheric drag, to slow a spacecraft down and enter orbit. The drag force from a single hyperbolic atmospheric pass provides the delta- $\mathrm{V}$ needed for orbit insertion. Atmospheric drag is a very efficient solution to change the velocity of a spacecraft because the total spacecraft mass is less coupled to the required delta- $\mathrm{V}$. This results in a drastic reduction in the propellant required, which can provide room for additional payload, such as science instrumentation, daughter craft, and atmospheric probes [1]. In addition, aerocapture can enable shorter transit times to planetary destinations because the spacecraft can arrive at a high relative velocity, unlike traditional propulsive capture where the propellant mass increases exponentially with the orbit insertion delta- $\mathrm{V}$ requirement. Aerocapture is especially useful for missions to the outer planets, where aerocapture can shorten cruise times by years, leading to a faster return of science data [2]. Aerocapture does introduce a potential packaging and configuration challenge as the spacecraft must be accommodated inside an aeroshell, but prior studies have shown this to be feasible.

The aerocapture maneuver is defined by three phases: approach, atmospheric deceleration, and

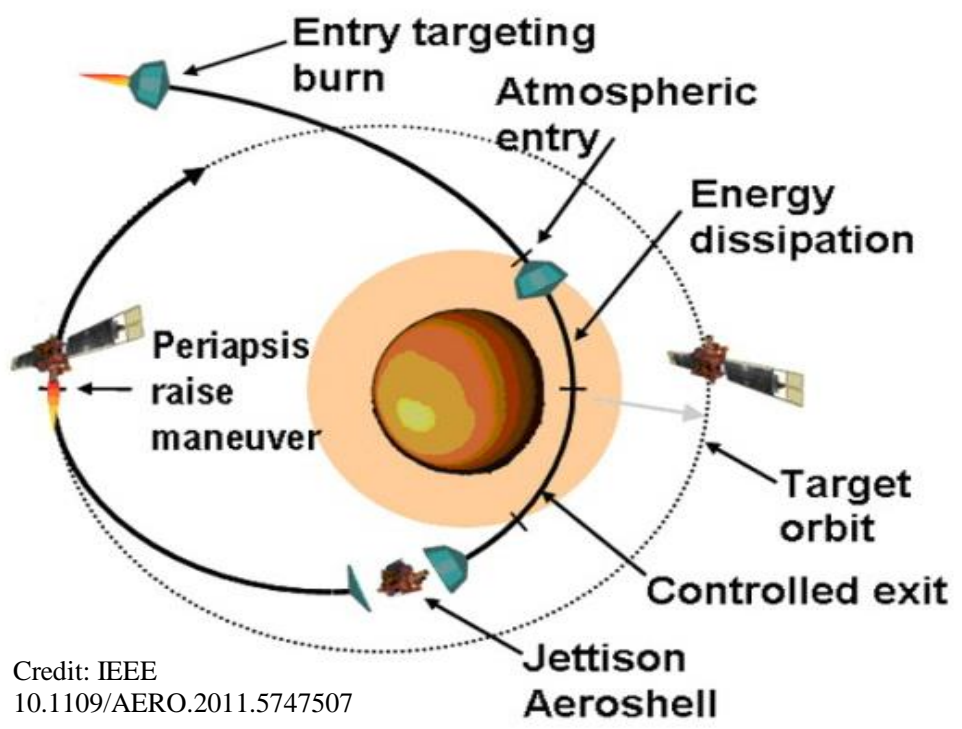

Figure 1: Aerocapture uses the drag from a single pass through the atmosphere to slow down and enter orbit, rather than a large burn from a propulsion system. 
orbital operations (Figure 1). On approach, the spacecraft targets a specific atmospheric interface state, similar to a lander mission. During the atmospheric deceleration phase, the aerocapture spacecraft uses a form of flight control to account for day-of-flight uncertainties. Many studies have been conducted to develop these control methods, which are most often grouped into two categories: lift modulation and drag modulation. Lift modulation is highly mature after demonstration on the Mars Science Laboratory (and soon to be on the Mars2020) mission [3], as well as the Apollo crew capsule's final guidance scheme. Drag modulation, the subject of more recent study, provides a control method that is potentially simpler to implement if the mission can tolerate less accurate final orbit targeting [4]. After the atmospheric pass, the spacecraft performs a small propulsive periapsis raise maneuver near apoapsis to enter the target orbit. Additional maneuvers can be used to adjust the orbit over time, if needed. While somewhat related, aerocapture is different from aerobraking because a single pass through the atmosphere is used to impart a significant delta- $\mathrm{V}$ on the spacecraft, rather than many repeated passes over a long period of time. This results in much larger propellant mass savings and enables faster cruise times, since aerobraking must perform a traditional propulsive orbit insertion maneuver to initially enter orbit.

Prior studies have highlighted the benefits that aerocapture technology can bring to large missions, and recent studies have focused on how the technology can enable small satellites to enter orbit [5]. Aerocapture is particularly well suited for SmallSat orbit insertion, due to the difficulty of designing and integrating a propulsion system to perform kilometers per second of delta-V on a small platform. Aerocapture presents a fast and efficient way for small platforms to enter orbit around planetary bodies and accomplish meaningful science objectives, allowing for new discoveries at a reduced cost.

Aerocapture technology provides a far-reaching capability for missions at destinations across the solar system, from SmallSat to Flagship class (Figure 2). It will enable a new class of SmallSat orbiters, allow high priority missions to carry more science payload to their destination, and open a new rapid transportation pathway to the outer solar system.

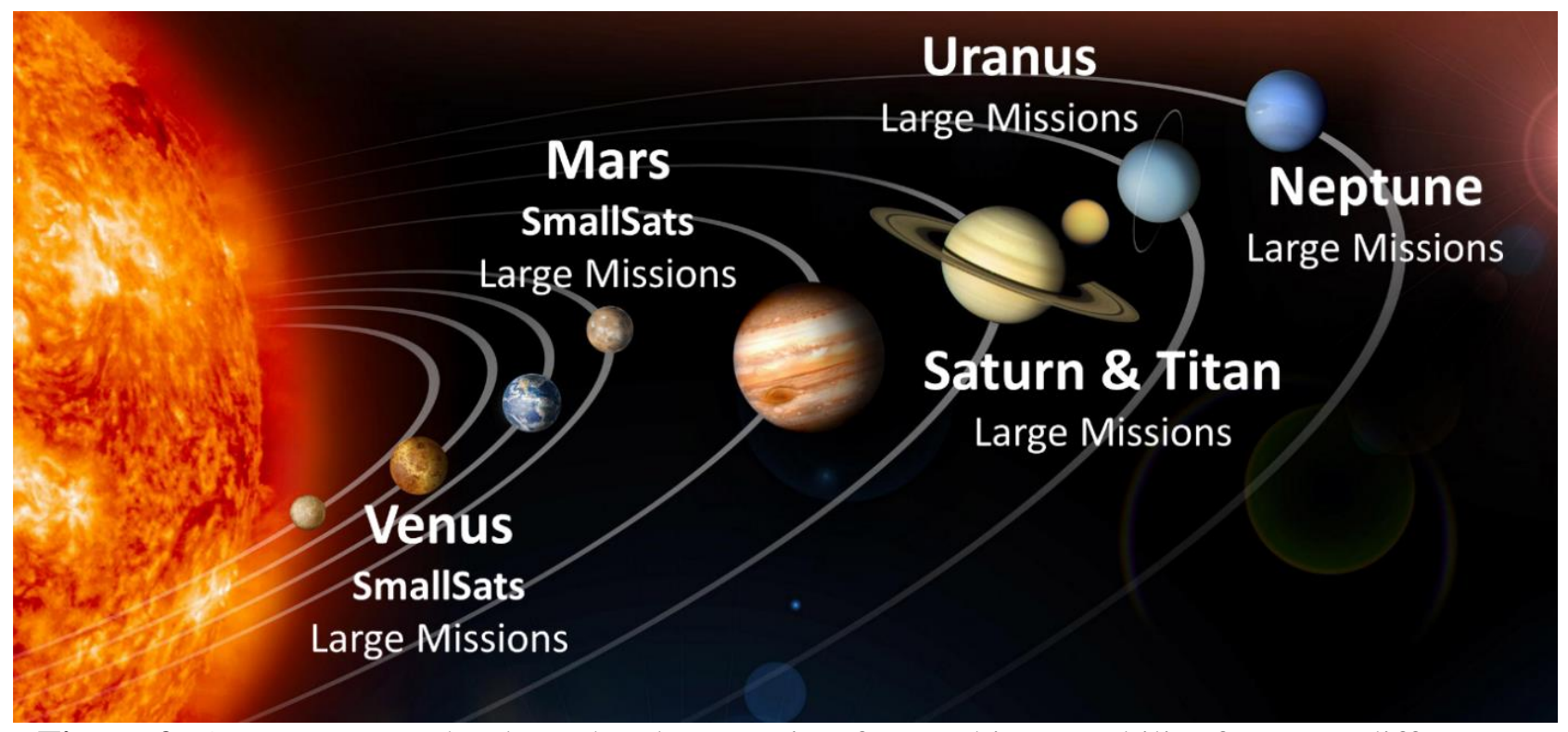

Figure 2: Aerocapture technology development is a far-reaching capability for many different mission classes across the solar system. 


\section{Aerocapture to Enable Planetary Small Satellite Science Missions (SIMPLEx)}

The last decade has seen an explosion of SmallSat missions that can make compelling scientific discoveries at a fraction of the cost of much larger missions. While these small spacecraft have mostly been confined to Earth orbit, in recent years they have branched out into the solar system. Most notably, the MarCO CubeSats launched with the Insight lander mission to provide critical relay capability during the entry, descent, and landing portion of the mission [6]. MarCO demonstrated the ability of a small spacecraft to accurately navigate through deep space, collect data, and send that data back to Earth. In the near future, 13 new CubeSats will make their way to cislunar space as secondary payloads on the Artemis 1 mission [7].

NASA has made their commitment to future small science missions clear, promising that an EELV Secondary Payload Adapter (otherwise known as an "ESPA ring") will be included on all future Science Mission Directorate (SMD) launches to allow for small spacecraft secondary payloads to utilize the excess launch mass capability of larger missions [8]. Many studies have confirmed the capability of small spacecraft to make Decadal-class science observations, including the 2018 Planetary Science Deep Space SmallSat Studies (PSDS3) [9]. NASA recently revived the Small Innovative Missions for Planetary Exploration (SIMPLEx) program, a proposal call to solicit SmallSat missions to launch on ESPA rings with primary SMD missions [10]. The expectation is that the SIMPLEx proposal solicitation will continue on an approximately yearly basis, giving ample opportunity for future small planetary science missions. While this undoubtedly provides an avenue for increased science exploration at lower cost, an additional benefit is the increased opportunities for new Principal Investigators to formulate and conduct a mission, preparing future leaders for the next decade and beyond.

While small spacecraft can clearly perform exceptional scientific exploration and there are ample opportunities for getting these spacecrafts on a trajectory to planetary destinations, there is still the fundamental challenge of developing an orbit insertion system compatible with the SmallSat rideshare paradigm, necessary to conduct a long-term science mission. SmallSats are highly mass and volume constrained, more often than not required to conform to strict standards that make them compatible for launch as a secondary payload with a large primary mission (e.g. maximum $180 \mathrm{~kg}$ and $61 \times 71 \times 97 \mathrm{~cm}$ for ESPA payloads) [10]. Accommodating a large propulsion system and the required propellant for orbit insertion at a planetary destination can be a significant challenge, or even impossible, depending on the delta- $\mathrm{V}$ required. MarCO, the only deep space SmallSat to date, had approximately $40 \mathrm{~m} / \mathrm{s}$ of delta-V [11], far from the required 1000 $-3000 \mathrm{~m} / \mathrm{s}$ required for reaching orbit at planets across the solar system.

By using the drag from a single pass through the atmosphere, aerocapture allows small spacecraft to enter orbit around other planets regardless of the delta-V required and within the constraints to allow for launch as a secondary payload. This is a far-reaching capability to enable a new class of small spacecraft science orbiters and expand the community's opportunities for new missions and exciting discoveries. Aerocapture enables any future launch targeting either another planet or performing a gravity assist flyby to carry aerocapture SmallSats that enter orbit and conduct their own science mission, or work in parallel with another mission. As examples, an outer planet mission performing a gravity assist flyby at Venus provides a launch opportunity for small Venus orbiters as secondary payloads, or a Mars mission can carry additional small aerocapture spacecraft to establish an orbital constellation to provide global coverage. These small aerocapture spacecraft may even be able to share a cruise stage with a primary mission, providing entry targeting capability and other spacecraft functions from the primary mission to the SmallSat [12]. 
SmallSat aerocapture has been the focus of significant research and technology development in the last five years, focused on developing a simple and costeffective atmospheric control method that is implementable on a small spacecraft within the constraints of a SIMPLEx-class mission [13]. The simplest form of drag modulation aerocapture is the single-stage discreteevent architecture (Figure 3) [4]. The spacecraft enters the atmosphere in a high-drag/low-ballistic coefficient configuration with its drag skirt deployed, and then transitions to a low-drag/high-ballistic coefficient by jettisoning the drag skirt. The timing of the drag skirt jettison event is determined by a control algorithm with input from an onboard inertial measurement unit. The algorithm monitors the deceleration of the vehicle and triggers the release of the drag skirt based on predicted atmospheric exit conditions that result in capture to the target orbit. By keeping the drag skirt attached longer, the system can slow down more, or it can be released sooner to slow down less. This form of drag modulation flight control provides the control authority needed for an interplanetary science mission with a simple enough implementation to be integrated as part of a small spacecraft.

Significant technology development prepares this form of aerocapture for infusion into small satellite missions. The deployable drag skirt technology is the Adaptable, Deployable Entry and Placement Technology (ADEPT). ADEPT employs an umbrella-like deployable structure with a "skin" that is a 3-D woven carbon fabric to serve as a thermal protection system (TPS) and as a structural surface that transfers aerodynamic deceleration forces to the underlying ribs. [14, 15]. The ADEPT structure is folded during launch and then deployed prior to atmospheric entry, enabling the entire flight system to be packaged into an ESPA compatible volume which meets SIMPLEx launch and configuration constraints. The ADEPT system is uniquely capable as it can tolerate the high heating environment at Venus, though other deployable drag area technologies, such as inflatable systems, are also possible. ADEPT benefits from significant recent development, culminating in a successful suborbital flight test which demonstrated key aspects of the technology, including the ability to deploy in space and withstand aerodynamic loading during reentry [16]. Other technology development efforts include dynamics simulations, computational fluid dynamics analysis, arc jet testing, and ballistic range test campaigns. This has given a comprehensive understanding of the aerocapture flight system and requirements, with the next step to prepare for technology demonstration and mission infusion to enable small satellite orbiter missions across the solar system.

\section{Aerocapture to Enable High Priority Large Missions (Discovery, New Frontiers and Flagship)}

Aerocapture technology does not only apply to small missions. In fact, even greater benefits can be realized for large missions of Discovery, New Frontiers, and Flagship class throughout the Solar System. The greater mass efficiency and reduced cruise time afforded by 
aerocapture, relative to fully-propulsive orbit insertion, can enable and enhance missions at multiple planetary destinations, particularly those that are high on the list of targets for exploration in the next decade.

This is perhaps most clear for the Ice Giants, Uranus and Neptune, which have only been visited once by the Voyager 2 flyby. The Ice Giants are continuously identified as high priority science targets for an orbiter and atmospheric probe. In addition to the planets themselves, the satellites and moons provide an exceptional opportunity for new discoveries. Missions to the Ice Giants are inherently difficult, however, due to their distance from Earth ( 20 AU for Uranus and $\sim 30$ AU for Neptune) which, for trajectories that reach the planets in a reasonable amount of time ( $<12$ years) requires a very high orbit insertion delta-V [17]. This results in propulsion system and propellant masses that can be prohibitive or impossible to accommodate. As aerocapture uses atmospheric drag to slow down, the system is relatively insensitive to the required delta- $\mathrm{V}$, which allows the spacecraft to devote more mass to science instrumentation or an additional spacecraft element, such as an atmospheric probe. The mission can also fly a faster trajectory that can remove years from the interplanetary flight time [2]. This decreased cruise time has a direct effect on the amount of science that can be returned, both because the science mission can start sooner and because the opportunities for extended missions increase due to the spacecraft spending more of its design lifetime at its destination. Such extended missions will be of great value in planning follow-up observations of new discoveries, as done by Cassini at Saturn.

Aerocapture can also enable and enhance missions to the Saturn system, including the high scientific priority ocean world targets Titan and Enceladus. The Titan atmosphere provides an ideal environment for aerocapture, due to the large scale height and relatively benign heating environment [17]. Titan is truly the gateway to the Saturn system, giving access to the moon itself [18], but also Saturn, the ring plane and, perhaps most importantly in the next decade, Enceladus [19]. Enceladus is considered a prime target for astrobiology exploration and the search for life elsewhere in the solar system. Flythrough of the South Pole plumes may provide opportunities for direct sampling of the ocean underneath its icy crust, and a lander mission would have the potential to revolutionize our understanding of life in the universe, especially if the liquid ocean could be reached. The challenge with an Enceladus mission is the same as any interplanetary space mission: getting delta-v. To reach Enceladus, a spacecraft must first slow down to enter Saturn orbit, then slow down again to reach Enceladus orbit, and finally slow down to reach the surface. Aerocapture at Titan can significantly reduce the propulsion and propellant mass requirements for the first Saturn orbit insertion maneuver, leading to a more mass efficient spacecraft to perform subsequent maneuvers to reach Enceladus [20]. The same aerocapture system can be used to enter Titan orbit, or enter a Saturn ring skimming orbit for science throughout the Saturn system.

While the benefits of aerocapture in terms of decreasing interplanetary cruise time are particularly relevant for missions to the outer planets, there can also be benefits at Venus [21] and Mars [22], especially in regards to increased mass efficiency of the flight system, enabling larger mass science payloads. Ultimately, aerocapture is applicable to future human Mars exploration; the technology has repeatably been identified as an enabler to bring the larger payload masses that will be required for humans to live and work on the planet [23].

In the past decade, significant technological progress has been made to prepare aerocapture technology for mission infusion. Particularly, the Mars Science Laboratory has demonstrated bank angle lift modulation hypersonic guidance at Mars (soon to be used a second time in the landing of the Mars 2020 rover) [3]. This atmospheric control method "steers" the spacecraft through the 


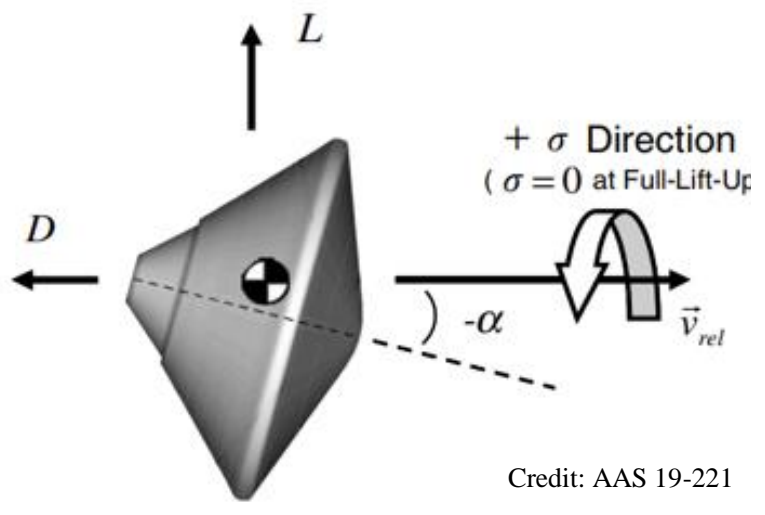

Figure 4: Hypersonic lift modulation control methods have been demonstrated at Mars and studied extensively for aerocapture missions. atmosphere by banking the spacecraft to reorient the lift vector (Figure 4). An advanced real-time hypersonic guidance algorithm, a numerical predictor-corrector, was also demonstrated on NASA's Exploration Flight 1 (EFT-1), similar to what would likely be used for aerocapture. This technology development progress has shown that we can use hypersonic guidance for planetary systems. Other key technology advancements include thermal protection systems to withstand the high heating environment at the Ice Giants [24] and optical navigation to allow accurate entry targeting [25]. All together, these developments indicate

that aerocapture is ready for use at the Ice Giants with high heritage aeroshell shapes flown since the 1960s [26]. Other more recent studies have also explored the applicability of drag modulation aerocapture to larger missions at Titan and the Ice Giants [27]. A companion paper to this white paper describes the applicability of aerocapture to Ice Giants missions in more detail [28].

\section{Aerocapture Technology Development and Mission Infusion Pathway}

Aerocapture technology provides a far-reaching capability for missions at destinations across the solar system, from SmallSat to Flagship class. Significant technology development and demonstration in the past decade has prepared high heritage aeroshells to be ready for infusion into large missions, particularly at the Ice Giants. In addition, research and focus on drag modulation control schemes has highlighted that this system can enable a new generation of small spacecraft science orbiters as secondary payloads, leading to increased science at a fraction of the cost.

Recent work has highlighted the opportunity to leverage the significant development in CubeSat and SmallSat flight system hardware for an extremely cost-effective technology demonstration of the drag modulation system in Earth orbit [29]. A small (less than 100 kilogram) aerocapture spacecraft would use either a secondary launch opportunity or a small launch vehicle to a highly elliptical orbit to perform a single atmospheric pass at relevant conditions to planetary entry. This would demonstrate the ability to target a desired orbit in the presence of day-of-flight uncertainties and validate the separation dynamics of the drag skirt from the spacecraft in hypersonic flight conditions (Figure 3). While this technology demonstration opportunity would be focused on the drag modulation control system, it would undoubtedly have an additional positive impact on the infusion of other aerocapture control methods into many missions, as well as demonstrate a number of key technologies and flight system development modes, including the ADEPT deployable aeroshell and relevant feed-forward to other small entry missions, such as small landers.

Aerocapture technology development and mission infusion has a clear path, beginning with the drag modulation SmallSat demonstration in Earth orbit, moving from there to many SmallSat and larger orbiters at planetary destinations, and ultimately culminating in a truly revolutionary Ice Giants mission and the human exploration of Mars. Aerocapture technology has the potential to change how we conduct planetary exploration for decades to come by improving science data return and enabling new mission architectures. 


\section{References}

[1] Hall, J. et al., "Cost-Benefit Analysis of the Aerocapture Mission Set", Journal of Spacecraft and Rockets, Vol. 42(2), 2005.

[2] Noca, M.A., and Bailey R.W., "Mission Trades for Aerocapture at Neptune", Jet Propulsion Laboratory, California Institute of Technology, Pasadena, CA, April 2006.

[3] G.F. Mendeck and L. C. McGrew, "Post-Flight EDL Entry Guidance Performance of the 2011 Mars Science Laboratory Mission." AAS 13-419.

[4] Zachary R. Putnam and Robert D. Braun, "Drag-Modulation Flight-Control System Options for Planetary Aerocapture," Journal of Spacecraft and Rockets, Vol. 51(1), 2014, pp. 139-150.

[5] Austin, A. et al., "SmallSat Aerocapture: Breaking the Rocket Equation to Enable a New Class of Planetary Missions", 70 ${ }^{\text {th }}$ International Astronautical Congress, 21-25 October 2019.

[6] A. Klesh, et al., "MarCO: Early Operations of the First CubeSats to Mars", 32 ${ }^{\text {nd }}$ Annual AIAA/USU Conference on Small Satellites, 2018.

[7] "Hitchhiking Into the Solar System: Launching NASA's First Deep-Space CubeSats", NASA Document NP2015-03-1483-HQ.

[8] Foust, J., "NASA bolsters SmallSat science programs", SpaceNews, August 62018.

[9] Mercer, C., "Planetary Science Deep Space SmallSat Studies (PSDS3)", 17 ${ }^{\text {th }}$ Meeting of the NASA Small Bodies Assessment Group, NASA GSFC, June 12-14 2017.

[10] NASA SIMPLEx Announcement of Opportunity, July 162019

[11] “JPL MarCO - Micro CubeSat Propulsion System”, Product Brief, VACCO, 2014

[12] Albert, S. et al., "Conceptual Development of AeroDrop: Aerocapture and Direct Entry for Two Spacecraft on a Common Approach Trajectory", AIAA Scitech 2020 Forum, Orlando, FL, USA, 2020.

[13] Austin, A. et al., "SmallSat Aerocapture to Enable a New Paradigm of Planetary Missions," 2019 IEEE Aerospace Conference, Big Sky, MT, USA, 2019, pp. 1-20.

[14] Venkatapathy, E.; et al, "Adaptive Deployable Entry and Placement Technology (ADEPT): A Feasibility Study for Human Missions to Mars", AIAA 2011-2608

[15] Smith et al., "Nano-ADEPT: An Entry System for Secondary Payloads," IEEE Aerospace Conference, Big Sky, MT, March 2015.

[16] Cassell, A. et al., "ADEPT Sounding Rocket One Flight Test Overview", AIAA Aviation 2019 Forum, Dallas, Texas, 17-21 June 2019.

[17] Lockwood et al., "Aerocapture Systems Analysis for a Neptune Mission", NASA TM-2006-214300

[18] Lockwood et al., "Aerocapture Systems Analysis for a Titan Mission”, NASA TM 2006-214273

[18] Lu, Y. et al., "Feasibility Assessment of Aerocapture for Future Titan Orbiter Missions," Journal of Spacecraft and Rockets, Vol. 55, No. 5, 2018, pp. 1125-1135.

[19] Lu, Y. et al., "Saturn and Enceladus Mission Design using Titan Aerogravity-Assist," Acta Astronautica, 2020.

[20] T. R. Spilker et al., "Analysis of architectures for the scientific exploration of Enceladus," 2009 IEEE Aerospace Conference, Big Sky, MT, 2009.

[21] Lockwood et al., "Systems Analysis for a Venus Aerocapture Mission”, NASA TM-2006-214291

[22] H.S. Wright et al., "Mars Aerocapture Systems Study", NASA TM-2006-214522

[23] A. Dwyer and T. Polsgrove, "Human Mars Entry, Descent and Landing Architecture Study: Phase 2 Summary", AIAA Space Forum, Orlando, Florida, 17-19 September 2018.

[24] Venkatapathy, E. et al., "Enabling Entry Technologies for Ice Giants Missions", Ice Giants Systems 2020, London, UK, 20-22 January, 2020.

[25] Martin-Mur, T. et. al, "The Next 25 Years of Deep Space Navigation,” 2008, AAS 08-057

[26] Girija, A.P et. al, "Aerocapture Performance Analysis for a Neptune Mission Using a Heritage Blunt-Body Aeroshell," 2019, AAS 19-815.

[27] Elliott, J. et al., "Mission Design Prospects for the Ice Giants", Ice Giants Systems 2020, London, UK, 20 22 January, 2020.

[28] Dutta, S. et. al, "Aerocapture as an Option for Ice Giants Missions", Planetary Science Decadal Survey 2023-2032 white paper, 2020.

[29] Werner, M. et al., "Development of an Earth Test to Demonstrate Viability of Mars Aerocapture", 55 AIAA Aerospace Sciences Meeting, Grapevine, Texas, 9-13 January 2017. 